\title{
InSCo-Gen: A MDD Tool for Web Rule-Based Applications
}

\author{
Joaquín Cañadas ${ }^{1}$, José Palma ${ }^{2}$, and Samuel Túnez ${ }^{1}$ \\ 1 Dept. of Languages and Computation, University of Almeria, Spain \\ $\{$ jjcanada, stunez\}@ual.es \\ 2 Dept. of Information and Communications Engineering, University of Murcia, Spain \\ jtpalma@um.es
}

\begin{abstract}
Rules and ontologies are widely used in software development since they provide semantic web applications with meaning and reasoning features. This demonstration paper presents InSCo-Gen, a Model-Driven Development (MDD) tool for Web rule-based applications, which constructs a functional Web architecture integrating a rule engine for reasoning tasks. Development process is based on conceptual models composed of ontologies and production rules. These models are the source for the MDD process, which automatically generates implementation of the Web application.
\end{abstract}

Keywords: MDD, Web Engineering, Rule-based systems.

\section{Introduction}

In Semantic Web context, development of rule languages and inference engines to provide Web information systems with reasoning capabilities is an important research topic. Production rules (if-conditions-then-actions) play a leading role in this aim, since they enable declarative representation of domain expert knowledge and business logic. Rule engines deal with rule bases and execute several inference methods for firing appropriate rules in order to deduct new information and achieve results [1].

This demo presents InSCo-Gen, an MDD tool for building Web rule-based applications. It supports the development process starting from the specification of conceptual models in Conceptual Modeling Language (CML), the formalism for knowledge representation defined by the CommonKADS methodology [2] for knowledge-based system development. A model-driven approach is then applied to produce the implementation of a functional Web architecture which integrates a rule engine for reasoning tasks. The tool was developed for supporting InSCo [3], a software development methodology that interweaves knowledge engineering and software engineering approaches.

This paper is organized as follows: Section 2 introduces the model-driven approach applied in InSCo-Gen. Next, the architecture of the Web rule-based application generated is described in Section 3. Finally, the main conclusions and future work are summarized. 


\section{The InSCo-Gen Model-Driven Approach}

The InSCo-Gen model-driven approach is based on conceptual models created in CML, a knowledge modeling language which entails simplified specification of ontology and production rules. A CML conceptual model is basically composed of two parts, domain schemas and knowledge bases. Domain concepts, binary relationships, rule types and value types (enumerated literals) are modeled in a domain schema. A knowledge base is composed of instances of concepts, instances of relationships called tuples, and instances of rules.

The main difference with other conceptual modeling languages is the possibility of modeling production rules through rule types and rule instances. A rule type describes the structure of a set of rules defining the concepts bound to the rule antecedent and to the rule consequent. Rule types are particularized into rule instances that represent specific, logical dependencies between concept attribute values of rule antecedent and consequent.

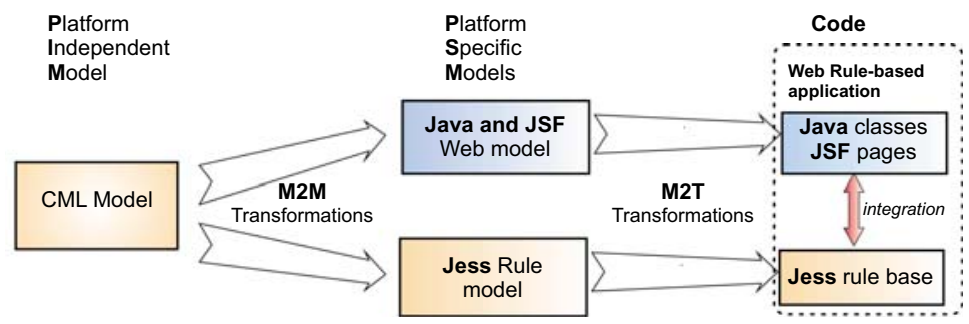

Fig. 1. MDD schema for Web rule-based system generation

Figure 1 shows a simplified schema of the two MDD processes implemented in InSCo-Gen. Two different results are found using a single CML model. On one hand, a Jess 4 ] rule base is generated, a text file that contains the rules converted to Jess syntax. On the other hand, a set of JavaBeans and JSP web pages, making up a JavaServer Faces (JSF) [5] architecture that integrates the Jess rule engine in a rich Web application.

Both MDD processes can be executed separately. Since the decision logic of rule-based applications may change frequently, the tool enables independent generation of a new rule base, which can be deployed in the Web application server without having to modify anything else in the architecture.

InSCo-Gen was developed using MDD tools provided by the Eclipse Modeling Project1. Models and metamodels are defined using EMF2 (Eclipse Modeling Framework). Three metamodels are needed, the CML metamodel for conceptual modeling, the Jess-Rule metamodel used for representing Jess platform-specific models and the Java/JSF metamodel used by Web-based specific models.

${ }^{1}$ http://www.eclipse.org/modeling/

${ }^{2}$ http://www.eclipse.org/modeling/emf/ 
Conceptual models based on the CML metamodel are created using the builtin EMF reflective editor. To improve model editing, the reflective editor was customized using Exeed (EXtended Emf EDitor) 6], a plugin which enables default icons and labels to be modified by using the Exeed annotations in the metamodel.

Two model-to-model (M2M) transformations are designed with ATL $3^{3}$ (Atlas Transformation Language). The first one (bottom flow in Fig. 1) maps a CML model to a Jess platform-specific model. The second one (top flow in Fig. 1) transforms a CML model into a Java/JSF Web specific model.

The outputs of both ATL transformations are the respective inputs of two model-to-text (M2T) transformations implemented in JET4 (Java Emitter Templates). As a result, InSCo-Gen automatically produces the application code. On one hand, source text files with Jess rules and facts, and on the other hand, the Web application components, the faces-config.xml and web.xml configuration files, the Java Beans for model classes, and a Jess-Engine Bean which uses the Jess Java API (Application Programming Interface) to integrate the rule engine into the architecture. Moreover, a set of JSP/JSF web pages are generated for the user interface. These pages are based on the RichFaces library [7, an open source framework that adds AJAX (Asynchronous JavaScript And XML) capability to JSF applications.

\section{Architecture of Web Rule-Based Applications}

Figure 2 shows the target architecture for Web rule-based applications generated by InSCo-Gen.
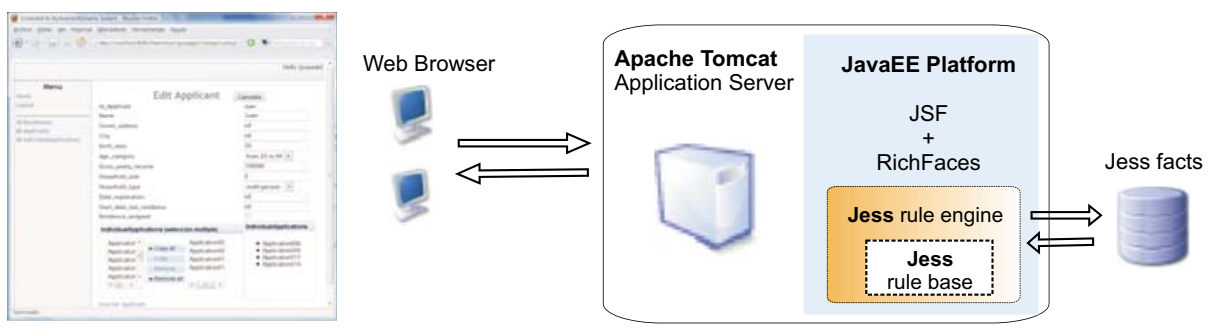

Fig. 2. Architecture of a Web rule-based application

The integrated rule engine manages the Jess rule base and the text file containing persistent instances of concepts, called facts. The Web application enables the user to perform four basic predetermined functions, create new instances, read the current list of instances, update and delete instances. That functionality makes the application generated a CRUD system (Create, Read, Update,

${ }^{3}$ http://www.eclipse.org/m2m/atl/

${ }^{4}$ http://www.eclipse.org/modeling/m2t/?project=jet 
Delete). In general, current tools for automatic generation of CRUD systems perform those operations on relational databases. The contribution of our approach is that CRUD operations are executed on the Jess rule engine working memory, enabling the inference mechanism to fire appropriate rules when necessary. The rule engine executes a forward-chaining inference mechanism to drive the reasoning process, firing the rules with conditions evaluated as true, and executing their actions to deduct new values or modify existing ones.

The use of both rules and AJAX technology improves the creation and edition of instances in the Web application. Since Web forms are implemented with AJAX RichFaces components, each single form value can be validated and submitted individually as it is entered. This facility entails the rule engine firing suitable rules and deducting new information that drives the instance creation or edition, for example, updating choice-field values.

\section{Conclusion and Future Work}

This demo presented InSCo-Gen 5, a real example of MDD applied to rich Web system development, incorporating a rule engine for reasoning tasks.

The tool is being evaluated within the development of a Web decision-support system for pest control in agriculture, which makes recommendations to growers and technicians about treatment for a specific pest or disease in grapes.

Our current work extends the tool with relational database facilities to provide a complete persistence layer. Also, a deliverable version of the tool as an Eclipse plugin will be available soon. Future incorporation of other semantic Web languages, such as OWL (Web Ontology Language) and SWRL (Semantic Web Rule Language) is planned.

\section{References}

1. Brachman, R.J., Levesque, H.J.: Knowledge representation and reasoning. Morgan Kaufmann, San Francisco (2004)

2. Schreiber, G., Akkermans, H., Anjewierden, A., de Hoog, R., Shadbolt, N., de Velde, W.V., Wielinga, B.: Knowledge Engineering and Management: The CommonKADS Methodology. The MIT Press, Cambridge (2000)

3. del Águila, I.M., Cañadas, J., Palma, J., Túnez, S.: Towards a methodology for hybrid systems software development. In: Proceedings of the Int. Conference on Software Engineering and Knowledge Engineering (SEKE), pp. 188-193 (2006)

4. Sandia Lab.: Jess, http://herzberg.ca.sandia.gov/jess/

5. Geary, D., Horstmann, C.S.: Core JavaServer Faces, 2nd edn. Prentice Hall, Englewood Cliffs (2007)

6. Kolovos, D.S.: Exeed: EXtended Emf EDitor - User Manual (2007), http://www.eclipse.org/gmt/epsilon/doc/Exeed.pdf

7. JBoss: RichFaces (2007), http://www.jboss.org/jbossrichfaces/

${ }^{5}$ This work is supported by two research projects, TIN2004-05694 funded by the Spanish MEC, and P06-TIC-02411 funded by the Junta de Andalucia. 